Check for updates

Cite this: RSC Adv., 2018, 8, 710

Received 13th September 2017 Accepted 18th December 2017

DOI: 10.1039/c7ra10174k

rsc.li/rsc-advances

\section{A novel triphenylacrylonitrile based AIEgen for high contrast mechanchromism and bicolor electroluminescence $\uparrow$}

\author{
Jiayou Hu, ${ }^{a}$ Bingli Jiang, ${ }^{\mathrm{b}}$ Yongyang Gong, (D) *a Yuanli Liu, ${ }^{a}$ Gang He, ${ }^{a}$ \\ Wang Zhang Yuan ${ }^{\star c}$ and Chun Wei ${ }^{\star a}$
}

A novel thermally stable and aggregation-induced emission (AIE) active compound, 2,2'-(([1,1'-biphenyl]4,4'-diylbis(phenylazanediyl))bis(4,1-phenylene))bis(3,3-diphenylacrylonitrile) (BP2TPAN) was synthesized through a $\mathrm{C}-\mathrm{N}$ coupling reaction between 2-(4-bromophenyl)-3,3-diphenylacrylonitrile (Br-TPAN) and $N, N^{\prime}$-diphenyl-1,4-phenylenediamine, under mild conditions using $\mathrm{Pd}(\mathrm{OAc})_{2}$ and $\mathrm{P}(t-\mathrm{Bu})_{3}$ as a catalyst. The BP2TPAN was characterized by nuclear magnetic resonance spectroscopy, high resolution mass spectrometry and elemental analysis. The thermal analysis showed that the glass transition and decomposition temperatures (5\% weight loss) are 96 and $414{ }^{\circ} \mathrm{C}$, respectively. The fluorescent emission peaks changes at 540 and $580 \mathrm{~nm}$ upon grinding were attributed to a transformation from crystal to amorphous occurring by altering the condensed state. The photoluminescence quantum yield and fluorescence lifetime of the as prepared and ground samples were 74.3 and $8.4 \%, 3.4$ and 5.1 ns, respectively. The difference of the luminous efficiency of before and after grinding samples indicates BP2TPAN has a high contrast more importantly, both doped and nondoped OLED devices emit different color, the doped one is highly efficient and its $L_{\max }, C E_{\max }, P E_{\max }$ and $E Q E$ are up to $15070 \mathrm{~cd} \mathrm{~m}^{-2}$, $11.0 \mathrm{~cd} \mathrm{~A}^{-1}, 7.5 \mathrm{~lm} \mathrm{~W}^{-1}$, and $3.1 \%$, respectively.

\section{Introduction}

Organic luminescent materials which show dynamically switchable solid-state emission in respect to mechanical stimuli such as shearing and grinding, have attracted increasing interest owing to their academic importance and promising applications in optical storage, mechanical sensors, displacement or deformation detectors, security paper, optical memory and optoelectronic devices. ${ }^{1-9}$ Efficient solid state emission is a key requirement for mechanochromic luminescent materials. However, conventional organic fluorescent molecules suffer from aggregation caused quenching (ACQ), ${ }^{10}$ which leads to poor solid state emission, and greatly restricts practical applications because luminescent materials are normally used in aggregated states, such as in OLEDs. Although intensive efforts have been made to reduce or completely eliminate the ACQ

${ }^{a}$ Key Laboratory of New Processing Technology for Non-ferrous Metals and Materials, Ministry of Education, Guangxi Key Laboratory of New Energy and Building Energy Saving, College of Materials Science and Engineering, Guilin University of Technology, Guilin 541004, China.E-mail: yygong@glut.edu.cn; 1986024@glut.edu. $\mathrm{cn}$

${ }^{b}$ College of Pharmacy, Guilin Medical University, Guilin 541004, China ${ }^{c}$ School of Chemistry and Chemical Engineering, Shanghai Jiao Tong University, Shanghai 200240, China. E-mail: wzhyuan@sjtu.edu.cn

$\dagger$ Electronic supplementary information (ESI) available: synthetic routes, TLC plates, NMR, HRMS, UV-Vis and CIE. See DOI: 10.1039/c7ra10174k effect through chemical or physical methods, such as introduction of bulky alicyclics, encapsulation by surfactants, or blending with transparent polymers, ${ }^{\mathbf{1 1}, 12}$ these approaches would inevitably damage their optoelectronic properties. Fortunately, in 2001, Tang and co-workers discovered a novel phenomenon from twisted siloles, an aggregation-induced emission (AIE), which is opposite to the ACQ effect. ${ }^{13}$ Namely, these compounds are practically nonluminescent in solutions, but become highly emissive in aggregated states. The AIE active molecules are highly fluorescent in the solid state, which is an essential requirement for mechanochromic materials.

The mechanochromic phenomenon have been explained as an alteration of molecular packing modes, such as crystal to amorphous form transformation, formation of excimers/ exciplex, or planarization under the action of forces. ${ }^{\mathbf{1 4}}$ Over the past few years, many novel twisted conformations AIE active mechanochromic materials have been developed by Chi, ${ }^{15}$ Park, ${ }^{16}$ Tian, ${ }^{17}$ Tang, ${ }^{18}$ et al. group, and the relationship between the molecular structure and the mehcanochromic luminescence has been evaluated. ${ }^{14}$ However, a few studies on AIE mechanochromic materials with its high thermal stability and high contrast have been reported. ${ }^{19}$ On the other hand, AIE compounds are ideal alternatives used for OLED applications. ${ }^{20}$ So far, however, many AIE compounds are mainly fabricated OLED devices with only one electroluminescent color. ${ }^{21-23}$ 


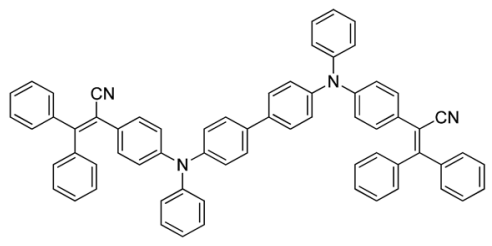

Scheme 1 Chemical structure of BP2TPAN

However, a few studies reported OLED devices with two or more electroluminescence emitting color based AIE compounds. ${ }^{24,25}$

Herein, a novel highly twisted conformation compound BP2TPAN with good thermal stability, containing a triphenylacrylonitrile (TPAN), as depicted in Scheme 1, was successfully synthesized by $\mathrm{C}-\mathrm{N}$ coupling between 2-(4-bromophenyl)-3,3diphenylacrylonitrile (Br-TPAN) and $N, N^{\prime}$-diphenyl-1,4-phenylenediamine (Fig. S1†).

BP2TPAN is AIE-active, and its photoluminescence quantum yield (PLQY) of as prepared samples ( $\Phi_{\text {asp }}$ ) up to $74.3 \%$. BP2TPAN solids also exhibit obvious mechanochromism behavior; upon grinding, the emission maxima of the as prepared powders were changed from 540 to $580 \mathrm{~nm}$. It's also showed good thermal stabilities, with degradation temperature ( $T_{\mathrm{d}}$, at which a sample loses $5 \%$ weight) and glass transition temperature $\left(T_{\mathrm{g}}\right)$ being are 414 and $96{ }^{\circ} \mathrm{C}$, respectively. More importantly, the doped and nondoped OLED devices show different emission color, which is rarely reported.

\section{Experimental}

\subsection{Materials}

Sodium tert-butoxide, sodium hydride (60 wt\% in mineral oil), palladium diacetate, $N, N^{\prime}$-diphenylbenzidine, were obtained from TCI (Shanghai) Development Co., Ltd. Benzophenone and 4-bromophenylacetonitrile were obtained from J\&K chemical Scientific Ltd. Tri-tert-butylphosphine ( $0.49 \mathrm{M}$ in toluene) was purchased from Puyang Huicheng Electronic Material Co., Ltd. Toluene was re-distilled under normal pressure from $\mathrm{CaH}_{2}$ under nitrogen immediately prior to used. THF was distilled from sodium benzophenone under nitrogen before used. The commercially available reagents were used without further purification.

\subsection{Characterization}

NMR spectrum was recorded on Bruker AMX-400 NMR spectrometer or Bruker AVANCE III HD600 $600 \mathrm{MHz}$ NMR instrument in deuterated solvent at room temperature. Highresolution mass spectrometry (HRMS) was performed on Bruker Daltonics ULTRAFLEXTREME MALDI-TOF/TOF mass spectrometer. UV-vis absorption and emission measurements were performed on a TU-1901 UV-vis spectrophotometer and a Perkin-Elmer LS 55 luminescence spectrometer, respectively. X-ray diffraction (XRD) measurements were carried out using Bruker D8 Advance TXS XRD with $\mathrm{Cu} \mathrm{K} \alpha$ radiation at room temperature. Thermogravimetric analysis (TGA) and differential scanning calorimetric (DSC) measurements were conducted on
TGA Q5000 V3.13 Build 261 instrument and Netzsch DSC 200 F3 under nitrogen at a heating and cooling rate of $5{ }^{\circ} \mathrm{C} \mathrm{min}^{-1}$, respectively. Emission quantum yields ( $\left.\Phi_{\mathrm{F}^{\prime} \mathrm{s}}\right)$ of BP2TPANs in solvents were estimated by using quinine sulfate $\left(\Phi_{\mathrm{F}}=54 \%\right.$ in $0.1 \mathrm{~N} \mathrm{H}_{2} \mathrm{SO}_{4}$ ) as standard, while solid-state efficiencies were determined using an integrating sphere. The ground-state geometries were optimized using the density functional with B3LYP hybrid functional at the basis set level of 6-31G(d). All calculation were performed using the Gaussian 09 package.

\subsection{OLED fabrication}

The device was fabricated by the following processes. First, ITOcoated glass substrates were cleaned successively using deionized water, acetone, and isopropanol in an ultrasonic bath and then dried in a drying cabinet followed by pretreatment with oxygen plasma. Then, the organic films of $N, N, N^{\prime}, N^{\prime}$-tetrakis(4methoxy-phenyl)benzidine (MeO-TPD), 4,4', $4^{\prime \prime}$-tri(9-carbazoyl) triphenylamine (TCTA), AN2TPAN, 4,7-diphenyl-1,10phenanthroline (Bphen), and 8-hydroxyquinolinatolithium (Liq) were deposited by thermal evaporation under a base vacuum of about $10^{-6}$ Torr. Finally, aluminum (Al) metal was evaporated in another vacuum chamber without breaking the vacuum. The thicknesses of the films were determined by quartz thickness monitors. The active area of the EL device, defined by the overlap of the ITO and the cathode electrode, was $3 \mathrm{~mm} \times 3 \mathrm{~mm}$. Current density-voltage and current efficiencycurrent density characteristics was measured with a computer controlled Keithley 2400 Source Meter and BM-7A Luminance Colorimeter. The electroluminescence spectrum was measured by a Labsphere CDS-610. All measurements were carried out under air at room temperature without device encapsulation.

\subsection{Synthesis}

Synthesis of 2-(4-bromophenyl)-3,3-diphenylacrylonitrile (Br-TPAN). Into a $100 \mathrm{~mL}$, two-necked, round bottom flask equipped with a condenser were placed $10 \mathrm{mmol}(1.83 \mathrm{~g})$ of benzophenone, $15 \mathrm{mmol}(603 \mathrm{mg}$ ) of $\mathrm{NaH}$ ( $60 \mathrm{wt} \%$ in mineral oil), and $50 \mathrm{~mL}$ of toluene under nitrogen. The mixture was stirred at $80{ }^{\circ} \mathrm{C}$ for $10 \mathrm{~min}$, then $11 \mathrm{mmol}(2.17 \mathrm{~g})$ of 4-bromobenzylnitrile in $10 \mathrm{~mL}$ of toluene was added drop-wise over 20 min while maintaining at $80^{\circ} \mathrm{C}$. The reaction was lasted for another $10 \mathrm{~h}$, cooling to room temperature, after solvent evaporation, $100 \mathrm{~mL}$ water and $50 \mathrm{ml}$ chloroform respectively, were added. The organic layer was collected and washed with brine three times. The organic phase was then dried over anhydrous $\mathrm{MgSO}_{4}$ and concentrated under vacuum. The crude product was purified by silica-gel column chromatography using petroleum ether/dichloromethane (DCM) as eluent (8/1-4/1). $3.05 \mathrm{~g}$ white solid was obtained in $84 \%$ yield. ${ }^{1} \mathrm{H}$ NMR $\left(400 \mathrm{MHz}, \mathrm{CDCl}_{3}\right)$, $\delta$ (TMS, ppm): $7.43(\mathrm{~m}, 5 \mathrm{H}), 7.34(\mathrm{~d}, 2 \mathrm{H}), 7.29(\mathrm{~m}, 1 \mathrm{H}), 7.22(\mathrm{t}$, $2 \mathrm{H}), 7.14(\mathrm{~d}, 2 \mathrm{H}), 7.01(\mathrm{~d}, 2 \mathrm{H}) \cdot{ }^{13} \mathrm{C}$ NMR $\left(100 \mathrm{MHz}, \mathrm{CDCl}_{3}\right)$, $\delta$ (TMS, ppm): 158.41, 140.12, 138.72, 133.80, 131.70, 131.25, $130.67,130.09,129.87,129.26,128.49,128.44,122.55,119.71$, 110.43.

Synthesis of BP2TPAN. Into a $50 \mathrm{~mL}$ two-necked round bottom flask were placed $1 \mathrm{mmol}(336 \mathrm{mg}) N, N^{\prime}$-diphenyl-1,4- 
phenylenediamine, $2.5 \mathrm{mmol}$ (900 mg) Br-TPAN, $3 \mathrm{mmol}$ (288 mg) of $t$-BuONa, $10 \% \mathrm{mmol}(22 \mathrm{mg}) \mathrm{Pd}(\mathrm{OAc})_{2}, 0.5 \mathrm{ml}$ of $\mathrm{P}(t-$ $\mathrm{Bu})_{3}(0.45 \mathrm{M}$ in toluene solution). The flask was evacuated under vacuum and flushed with dry nitrogen for three times. Then $30 \mathrm{~mL}$ fresh toluene was injected. Then the mixtures were heated to reflux under stirring for $36 \mathrm{~h}$. Upon cooling to room temperature, the mixture was added $5 \mathrm{~g}$ silica (200-300 mesh), then, mixing uniform. Soxhlet extractor $(250 \mathrm{~mL})$ was placed $30 \mathrm{~g}$ silica (200-300 mesh), and the mixing mixture was added, the target compound BP2TPAN was extraction by $500 \mathrm{~mL}$ chloroform. Solvent was reduce evaporation to $100 \mathrm{~mL}$, filtrate and filters wash with $20 \mathrm{~mL}$ chloroform three times, $621 \mathrm{mg}$ yellow solid obtained in $69 \% .{ }^{1} \mathrm{H}$ NMR (600 MHz, THF-d 8 , $\delta$ ) 7.58-7.39 (m, 14H, Ar-H), 7.32-7.02 (m, 26H, Ar-H), 6.98-6.88 (m, 4H, Ar-H), 6.68-6.58 (m, 2H, Ar-H); HRMS (MALDI, $m / z)$ : [M $+\mathrm{H}]$ calcd for $\mathrm{C}_{66} \mathrm{H}_{46} \mathrm{~N}_{4}, 894.37225$; found 894.28727. Elemental analysis calcd (\%): C, $88.65 \%$; H, 5.18\%; N, 6.26\%; found: C $88.08 \%$, H $5.33 \%$, N 6.31\%.

\section{Results and discussion}

\subsection{Synthesis}

BP2TPAN was synthesized according to the route shown in Fig. S1 (ESI $\dagger$ ). First, Br-TPAN was synthesized according to the literature procedure through a Knoevenagel condensation reaction between 4-bromophenylacetonitrile and benzophenone. ${ }^{26}$ Then, the target molecule, desirable compounds of BP2TPAN, was obtained in good yields by Buchwald-Hartwig $\mathrm{C}-\mathrm{N}$ cross-coupling reaction between Br-TPAN and $N, N^{\prime}$ diphenyl-1,4-phenylenediamine, using $\mathrm{Pd}(\mathrm{OAc})_{2}$ and $(t-\mathrm{Bu})_{3} \mathrm{P}$ as catalysts under mild conditions. ${ }^{27}$

BP2TPAN was characterized by standard spectroscopic methods, from which satisfactory analysis data corresponding to its molecular structures was obtained (see Fig. S2-4 ESI $\dagger$ for detail). For example, thin layer chromatography (TLC) plates indicated high purity of the isolated compounds in 1:1 petroleum ether-chloroform solvent mixture, as only a single spot was obtained for each compound (Fig. S2, ESI $\dagger$ ). HRMS (Fig. S4 ESI $\dagger$ ) peak $[\mathrm{M}]^{+}$at $\mathrm{m} / z \quad 894.28727$ (calcd 894.37225) confirms the formation of expected adducts. Moreover, elemental analysis results of C 88.08\%, H 5.33\%, and N 6.31\% are highly consistent with their theoretical values. Results altogether indicates that BP2TPAN was sufficiently pure.

\subsection{AIE}

Our previous work have confirmed that TPAN is a typical crystallization-induced emission luminogens, ${ }^{28}$ which could yielded new AIE activated compounds when combined with aniline, diphenylamine, triphenylamine and carbazole et $a l^{2,8,24,27,28}$ It is therefore envisioned that BP2TPAN is also an AIEgen. BP2TPAN shows two absorption peaks at 340 and $394 \mathrm{~nm}$ in THF (Fig. S5, ESI $\dagger$ ). The former is attributable to the $\pi-\pi^{*}$ transition, the latter corresponds to the intramolecular charge transfer (ICT) transition. To verify AIE activity of BP2TPAN, it was slightly solubilized in organic solvents (THF), no visible emission was observed. However, as prepared (Asp) solid powder, it emits strong yellow light at $540 \mathrm{~nm}$ with quantum efficiency of $74.3 \%$, indicating that it is AIE-active. Emission spectra of BP2TPAN in THF and THF/water mixtures were also measured. Water was chosen because it is a typical nonsolvent for BP2TPAN, in which BP2TPAN molecules will be aggregated. When the water fraction $\left(f_{\mathrm{w}}\right)$ is $\leq 60 \%$, only weak signals were recorded, since the molecules were dissolved in the mixture. However, when $f_{\mathrm{w}}$ is increased to $70 \%$, the emission intensity was swiftly boosted because of the molecular aggregation, giving a strong yellow emission at $560 \mathrm{~nm}$ (Fig. 1). Further addition of water continuously strengthened the emission intensity. In the $10: 90 \mathrm{THF}-$ water mixture, the emission was remarkably enhanced compared to that in THF (Fig. 1). The AIE nature of BP2TAPN also visible from the emission contrast of BP2TAPN in THF and 10 : 90 THF-water mixture (Fig. 1B). To quantitatively evaluate the AIE effect, PLQY of the luminogens in both solution $\left(\Phi_{\text {soult }}\right)$ and as-prepared solid states were determined. The $\Phi_{\text {soult }}$ values of BP2TPAN in THF are as low as $0.1 \%$, thus confirming that it is practically non-luminescent nature in solvents. However, the values for as prepared solids increased to $74.3 \%$, indicating AIE characteristic.

In order to better understand the nature of AIE and the geometry of BP2TPAN, quantum chemical optimization of their energy levels based on DFT/B3LYP/6-31G(d) was conducted. The optimized geometries and HOMO/LUMO plots of BP2TPAN are illustrated in Fig. 2. The molecule adopt highly twisted propeller-like non-planar conformations, which are favorable
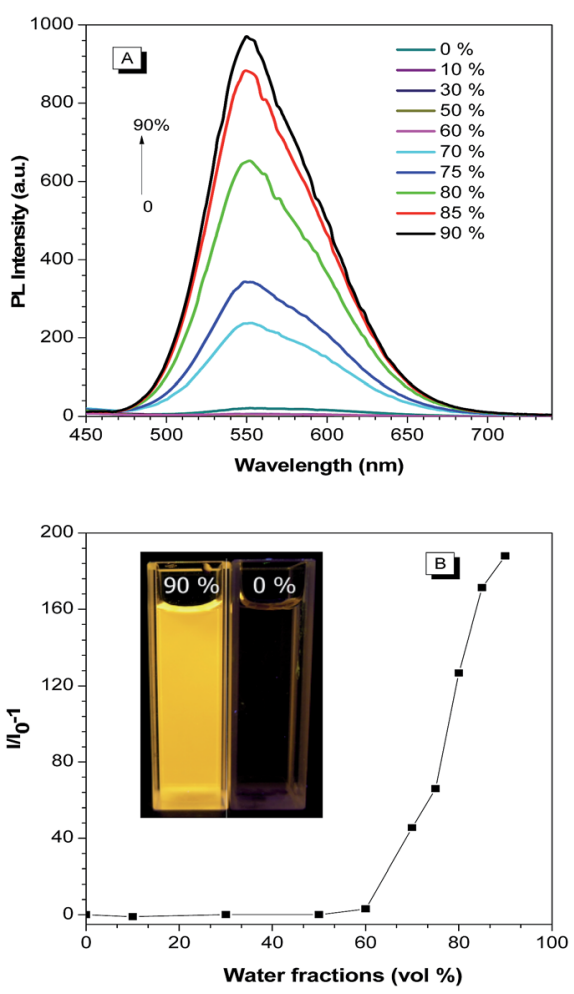

Fig. 1 (A) PL spectra of BP2TPAN in THF and THF/water mixtures. (B) Plots of PL peak intensity $v s$. water fraction $\left(f_{\mathrm{w}}\right)$. Concentration $=5 \mu \mathrm{M}$; excitation wavelength $=400 \mathrm{~nm}$. The inset graphs are the solution of BP2TPAN in THF $\left(f_{\mathrm{w}}=0 \%\right)$ and its suspensions in THF/water mixture with $f_{\mathrm{w}}=90 \%$ under $365 \mathrm{~nm}$ UV light illumination. 

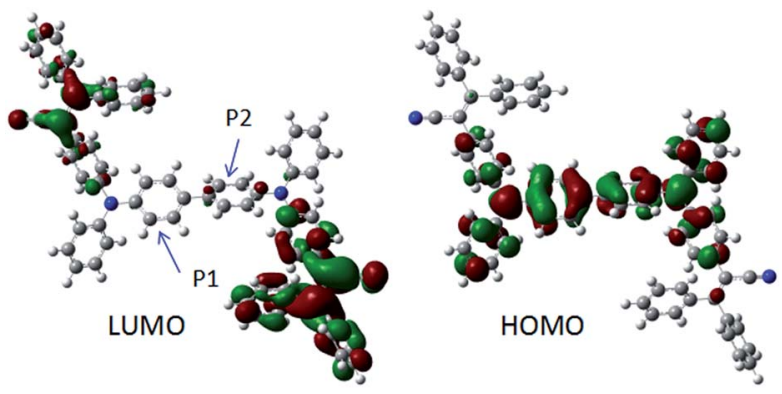

Fig. 2 Optimized geometries and molecular orbital amplitude plots of HOMO and LUMO energy levels of BP2TPAN.

for active intramolecular rotations of multiple phenyls in solutions, thereby powerfully dissipating the excitons energy and making them non-emissive in solvents. When aggregated as suspensions, powders, thin solid films, on one hand, the intramolecular rotations are impeded; on the other hand, the propeller-like configurations prevent the formation of detrimental excimers or exciplexes, thereby generating boosted emissions. Notably, the dihedral angles of the adjacent phenyl rings $(P 1$ and $P 2)$ is $\approx 20^{\circ}$, the non-planar conformations of biphenyl ( $P 1-P 2)$ decreased conjugated degree. Moreover, the electron clouds of the LUMO levels for BP2TPAN are mainly located on the electron-accepting TPAN peripheries; however, those of HOMO levels are dominated by orbitals from the electron-donating triphenylamine units. Generally, such electron distribution imparts an intrinsic intramolecular charge transfer property to dye molecules. This intramolecular charge transfer might balances hole and electron mobility in OLED devices and improve device performance.

\subsection{Mechanochromic behavior}

The remarkably twisted conformations and high PLQY of BP2TPAN make it potentially promising as mechanochromic luminogen with a high contrast. Upon grinding in an agate mortar with a pestle, the emission color of the as prepared solid changes from yellow to orange (Fig. 3A), with its emission maximum red shifted from 540 to $580 \mathrm{~nm}$ and the (Fig. 3B). When the ground powder annealed at $120^{\circ} \mathrm{C}$ under nitrogen for 10 min or fumed with chloroform vapor, the emission peaks restored to $542 \mathrm{~nm}$ (Fig. 3B).

To get more insights into the mechanism, XRD measurement of the as prepared, ground, and annealed samples were performed. As shown in Fig. 3C, the as prepared BP2TPAN display sharp and intense reflections at $2 \theta \approx 8.6^{\circ}$ and $19.5^{\circ}$, which indicate their well-defined crystalline order. However, the diffraction curves of the ground sample do not show any noticeable reflections in low intensity, indicating an amorphous structure. The amorphous sample, when annealed at $120{ }^{\circ} \mathrm{C}$ in $\mathrm{N}_{2}$ atmosphere, the diffraction peaks which coincided with those in the as prepared solids emerge again, indicating recovery of some crystalline order. These results also indicate that the interchange between crystalline-amorphous packing modes causes the mechanochromism effect in BP2TPAN.
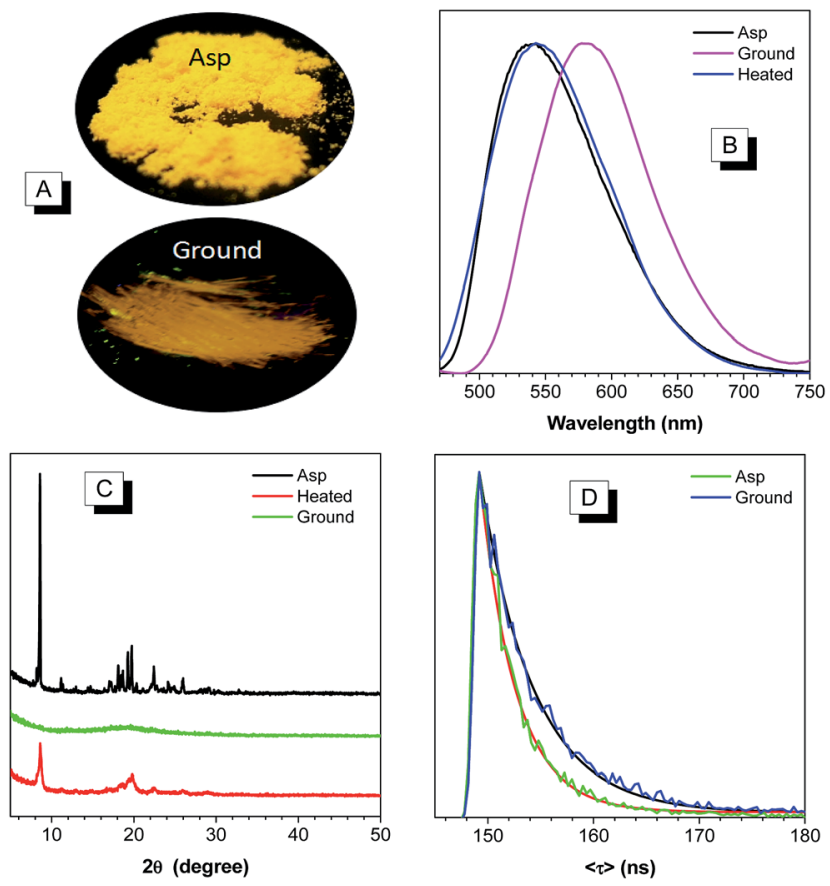

Fig. 3 (A) The photographs of as prepared BP2TPAN and its ground samples under $365 \mathrm{~nm}$ UV irradiation. (B and C) Are emission spectra and XRD patterns of BP2TPAN under different condensed states. (D) Fluorescence lifetime of BP2TPAN and its ground sample.

Although mechanochromic materials with switchable emission colors are reported in the literature, use external force to manipulate the fluorescent quantum yield is seldom reported. ${ }^{29}$ For BP2TPAN, PLQY of its the ground sample is lower than those as prepared samples, decrease of PLQY from $74.3 \%$ to $8.4 \%$ was observed. This result is consistent with the results from the naked eye observation, and indicating BP2TPAN has high contrast. This phenomenon may be attributed to crystals that readily break, trigger planarization of molecular conformation under external pressure, as well as the formation of excimers. Theoretically, excimer formation would promote fluorescence lifetime and decrease photoluminescence quantum yield. ${ }^{18}$ Herein the fluorescence lifetime values of the as prepared and ground amorphous solids of BP2TPAN are determined as 3.4 and $5.1 \mathrm{~ns}$ (Fig. 3D), respectively. The increased lifetime and decrease photoluminescence quantum yield of the ground sample suggested ground samples forms of excimers upon mechanical stimuli.

\subsection{Thermal stability}

Excellent thermal stability is highly desired for the device fabrication and optoelectronic applications of the molecular conjugates. Particularly, $T_{\mathrm{g}}$ of an organic luminophore is one of the most important factors that influence the device stability and lifetime. ${ }^{19}$ When device is heated above $T_{\mathrm{g}}$ of the organic luminophore, an irreversible failure can occur. Therefore, we checked the thermal property by measuring TGA and DSC of BP2TPAN. As shown in Fig. 4, BP2TPAN has high thermal stability with its $T_{\mathrm{d}}$ (defined as the temperature at which 

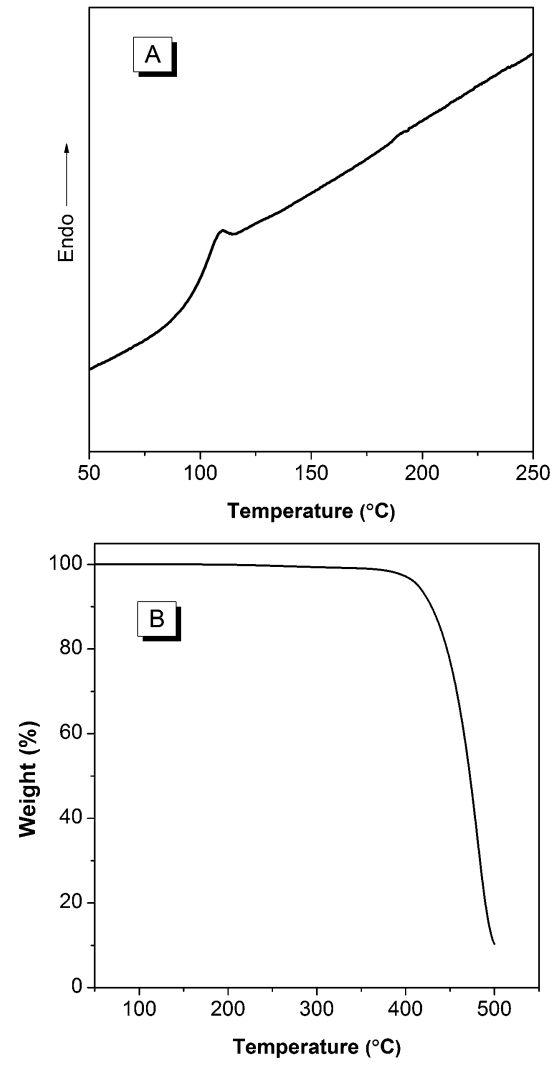

Fig. 4 (A) DSC (second heating cycle) and (B) TGA thermogram of BP2TPAN recorded under nitrogen at scan rates of $5{ }^{\circ} \mathrm{C} \mathrm{min}^{-1}$, respectively. a sample loses its $5 \%$ weight) value $414{ }^{\circ} \mathrm{C}$. DSC analysis reveals the $T_{\mathrm{g}}$ of BP2TPAN is $96{ }^{\circ} \mathrm{C}$, thus suggesting their exceptional thermal stability. Such outstanding thermal properties and efficient solid-state emissions render its application in optoelectronic devices.

\subsection{OLED}

The high solid-state emission efficiency and good thermal properties of BP2TPAN prompted us to explore their potential application in OLED devices. We first fabricated multilayer nondoped OLEDs with a general device configuration of ITO/NPB $(50 \mathrm{~nm}) / \mathrm{BP} 2 \mathrm{TPAN}(20 \mathrm{~nm}) / \mathrm{Bphen}(40 \mathrm{~nm}) / \mathrm{LiF} \quad(1 \mathrm{~nm}) / \mathrm{Al}$ $(100 \mathrm{~nm})$, where NPB and Bphen were chosen as hole- and electron-transporting layers, respectively. The electroluminescence (EL) spectrum, current density-voltage-luminance characteristics, current efficiency, power efficiency and external quantum efficiency of the devices are shown in Fig. 5 and $56 \dagger$ and summarized in Table 1 . The nondoped devices (I), emit orange light with EL maxima $\left(\lambda_{\mathrm{EL}}\right)$ at $570 \mathrm{~nm}$ which are rather close to the PL emissions of their ground powders $(580 \mathrm{~nm})$. The device performance was moderate, with turn on voltages $\left(V_{\mathrm{on}}\right)$, maximum luminance $\left(L_{\max }\right)$, current efficiency $\left(\mathrm{CE}_{\max }\right)$, power efficiency $\left(\mathrm{PE}_{\max }\right)$, external quantum efficiency (EQE), and commission Internationale de L'Eclairage (CIE) being $4.8 \mathrm{~V}$, $925 \mathrm{~cd} \mathrm{~m}^{-1}, 2.9 \mathrm{~cd} \mathrm{~A}^{-1}, 0.84 \mathrm{~lm} \mathrm{~W}^{-1}, 1.1 \%$ and CIE $(0.48,0.51)$, respectively. Moreover, interestingly, when BP2TPAN doped into 2-methyl-9,10-di(2-naphthyl)anthracene (MADN) at a doping level of $3 \%$ (wt\%) as an emitting layer, the device performance was dramatically improved by several times with
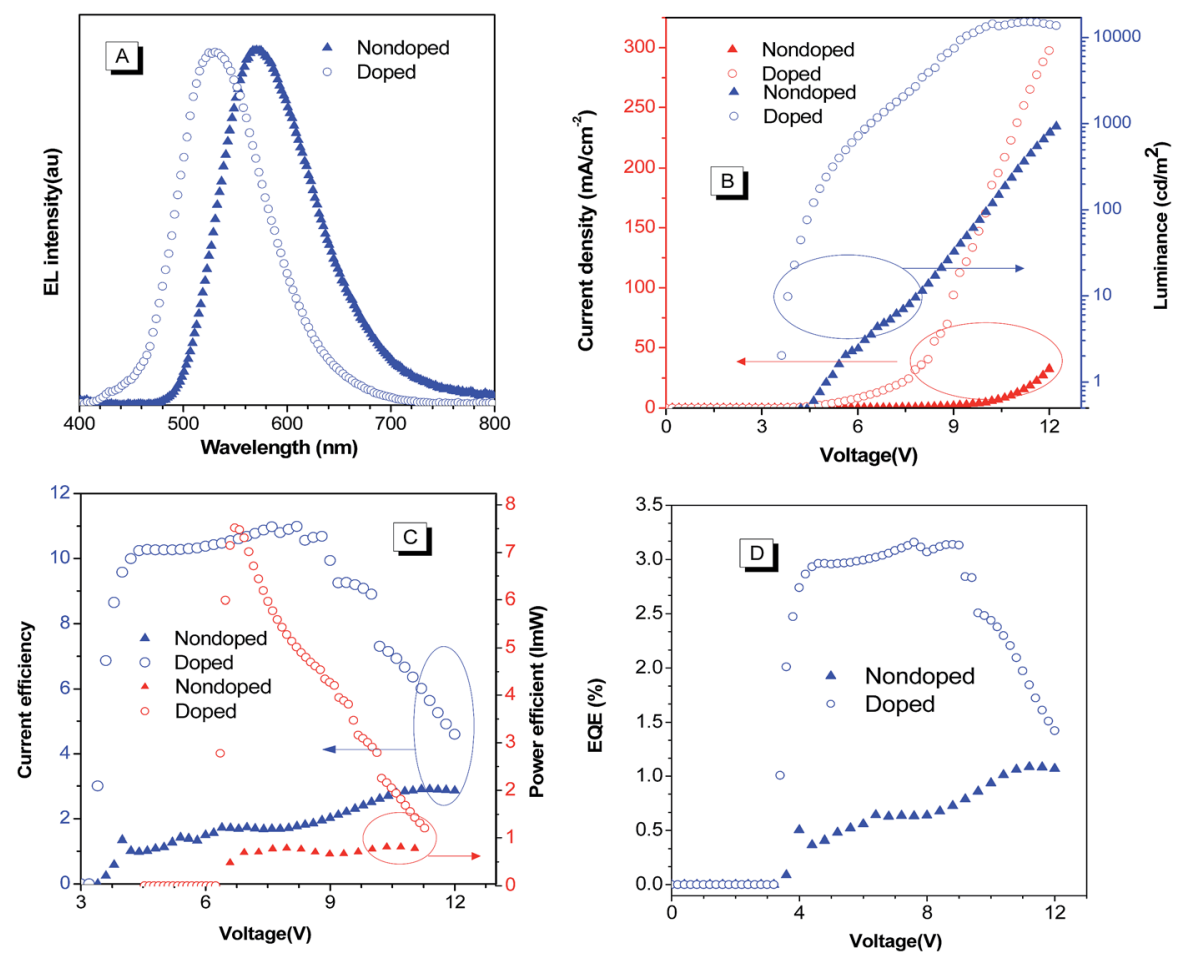

Fig. 5 (A) EL spectra of BPA2TPAN doped and nondoped OLED devices (B) current efficiency-voltage-luminance, (C) current efficiency, power efficiency, and (D) external quantum efficiency vs. driving voltage of the devices with a general configuration of ITO/NPB/X/Bphen/LiF/Al, $X=$ BP2TPAN (device I), BP2TPAN (3\%)-MADN (device II). 
Table 1 OLED device performances of BP2TPAN ${ }^{a}$

\begin{tabular}{|c|c|c|c|c|c|c|c|}
\hline Device & $V_{\text {on }}, \mathrm{V}$ & $\lambda_{\max }, \mathrm{nm}$ & $L_{\max }, \mathrm{cd} \mathrm{m}^{-2}$ & $\mathrm{CE}_{\max }, \mathrm{cd} \mathrm{A}^{-1}$ & $\mathrm{PE}_{\max }, \operatorname{lm} \mathrm{W}^{-1}$ & EQE, \% & $\mathrm{CIE}, x, y$ \\
\hline I & 4.8 & 570 & 925 & 2.9 & 0.84 & 1.1 & $0.48,0.51$ \\
\hline II & 3.2 & 530 & 15070 & 11.0 & 7.5 & 3.1 & $0.32,0.55$ \\
\hline
\end{tabular}

${ }^{a}$ Device configuration: ITO/NPB (50 nm)/X (20 nm)/Bphen (40 nm)/LiF (1 nm)/Al (100 nm); X = BP2TPAN (device I), BP2TPAN (3\%)-MADN (device II); abbreviations: $V_{\mathrm{on}}=$ turn on voltages, $\lambda_{\max }=\mathrm{EL}$ peak, $L_{\max }=$ maximal luminance, $\mathrm{PE}_{\max }=$ maximal power efficiency, $\mathrm{CE}_{\max }=$ maximal current efficiency, $\mathrm{EQE}=$ maximal quantum efficiency, $\mathrm{CIE}=$ Commission Internationale de L'Eclairage.

$V_{\text {on }}, L_{\max }, \mathrm{CE}_{\max }, \mathrm{PE}_{\max }, \mathrm{EQE}$ and CIE are $3.2 \mathrm{~V}, 15070 \mathrm{~cd} \mathrm{~m}^{-1}$, $11.0 \mathrm{~cd} \mathrm{~A}^{-1}, 7.5 \mathrm{~lm} \mathrm{~W}^{-1}, 3.1 \%$ and CIE (0.32, 0.55) (Fig. 5, Table 1 and Fig. S6 ESI $\dagger$ ), respectively. Such considerably enhanced EL performance for the doped devices should be ascribed to the better matching of the energy gaps of each layer and thus a more effective charge balance in the devices. These results also suggest the feasibility of regulating the EL color through device technics.

Indeed, the $\lambda_{\mathrm{EL}}$ values of the resulting doped and nondoped OLED devices were slightly blue shifted about $10 \mathrm{~nm}$ compared to ground and as prepared samples. This may be caused by under thermal evaporation, the molecule adopted more twisted configuration, and decreased conjugation length made EL spectrum slightly blue shifted. ${ }^{30,31}$

\section{Conclusions}

In summary, a novel triphenylacrylonitrile based BP2TPAN, whose highly twisted configurations are AIE luminogens, was successfully synthesized and applied for the mechanochromism and OLED applications. This AIE luminogens show high thermal stabilities, with their $T_{\mathrm{d}}$ and $T_{\mathrm{g}}$ values are 414 and $96{ }^{\circ} \mathrm{C}$, respectively. The emission peaks, photoluminescence quantum yields and lifetimes of BP2TPAN and its ground sample are 540 and $580 \mathrm{~nm}, 74.3$ and $8.4 \%, 3.4$ and $5.1 \mathrm{~ns}$, respectively. The non-optimized OLED non-doped device of the luminogens with $V_{\text {on }}, L_{\max }, \mathrm{CE}_{\max }, \mathrm{PE}_{\max }$ and $\mathrm{EQE}$ are $4.8 \mathrm{~V}$, $925 \mathrm{~cd} \mathrm{~m}^{-2}, 2.9 \mathrm{~cd} \mathrm{~A}^{-1}, 0.84 \mathrm{~lm}^{-1}$, and $1.1 \%$, respectively, giving yellow light $(570 \mathrm{~nm})$ EL with moderate efficiencies. Notably, the doped OLEDs devices emit significantly blue shifted yellow emissions, (530 $\mathrm{nm}$ ) with considerably enhanced performance, whose $V_{\text {on }}, L_{\max }, \mathrm{CE}_{\max }, \mathrm{PE}_{\max }$ and EQE are $3.2 \mathrm{~V}$, $15070 \mathrm{~cd} \mathrm{~m}^{-2}, 11.0 \mathrm{~cd} \mathrm{~A}^{-1}, 7.5 \mathrm{~lm} \mathrm{~W}^{-1}$, and 3.1\%, respectively. The AIE feature, high solid-state efficiency, mechanochromic properties, excellent thermal stability, and bicolor EL render BP2TPAN as a highly promising candidate for versatile optoelectronic applications.

\section{Conflicts of interest}

There are no conflicts to declare.

\section{Acknowledgements}

This work was financially supported by the National Natural Science Foundation of China (51603050), the Natural Science
Foundation of Guangxi (2016GXNSFBA380064), the Open Project Foundation of Guangxi Ministry-Province JointlyConstructed Cultivation Base for State Key Laboratory of Processing for Non-ferrous Metal and Featured Materials (15-KF10) and Guangxi Key Laboratory of New Energy and Building Energy Saving (16-J-21-3).

\section{Notes and references}

1 G.-F. Zhang, H. Wang, M. P. Aldred, T. Chen, Z.-Q. Chen, X. Meng and M.-Q. Zhu, Chem. Mater., 2014, 26, 4433-4446.

2 W. Z. Yuan, Y. Tan, Y. Gong, P. Lu, J. W. Lam, X. Y. Shen, C. Feng, H. H. Y. Sung, Y. Lu, I. D. Williams, J. Z. Sun, Y. M. Zhang and B. Z. Tang, Adv. Mater., 2013, 25, 2837-2843.

3 Q. Benito, X. F. Le Goff, S. Maron, A. Fargues, A. Garcia, C. Martineau, F. Taulelle, S. Kahlal, T. Gacoin, J.-P. Boilot and S. Perruchas, J. Am. Chem. Soc., 2014, 136, 11311-11320.

4 Y. Q. Dong, J. W. Lam and B. Z. Tang, J. Phys. Chem. Lett., 2015, 6, 3429-3436.

5 D. Yang, S. Ye and J. Ge, Adv. Funct. Mater., 2014, 24, 31973205.

6 Z. Ma, Z. Wang, X. Meng, Z. Ma, Z. Xu, Y. Ma and X. Jia, Angew. Chem., Int. Ed., 2016, 55, 519-522.

7 Z. Wu, J. Liu, Y. Gao, H. Liu, T. Li, H. Zou, Z. Wang and K. Zhang, J. Am. Chem. Soc., 2015, 137, 12906-12913.

8 Y. Lu, Y. Tan, Y. Gong, H. Li, W. Yuan, Y. Zhang and B. Z. Tang, Chin. Sci. Bull., 2013, 58, 2719-2722.

9 T. Seki, Y. Takamatsu and H. Ito, J. Am. Chem. Soc., 2016, 138, 6252-6260.

10 S. W. Thomas, G. D. Joly and T. M. Swager, Chem. Rev., 2007, 107, 1339-1386.

11 S. F. Lim, R. H. Friend, I. D. Rees, J. Li, Y. Ma, K. Robinson, A. B. Holmes, E. Hennebicq, D. Beljonne and F. Cacialli, Adv. Funct. Mater., 2005, 15, 981-988.

12 B. S. Gaylord, S. Wang, A. J. Heeger and G. C. Bazan, J. Am. Chem. Soc., 2001, 123, 6417-6418.

13 J. Luo, Z. Xie, J. W. Lam, L. Cheng, H. Chen, C. Qiu, H. S. Kwok, X. Zhan, Y. Liu, D. Zhu and B. Z. Tang, Chem. Commun., 2001, 1740-1741.

14 Z. Chi, X. Zhang, B. Xu, X. Zhou, C. Ma, Y. Zhang, S. Liu and J. Xu, Chem. Soc. Rev., 2012, 41, 3878-3896.

15 T. Yu, D. Ou, L. Wang, S. Zheng, Z. Yang, Y. Zhang, Z. Chi, S. Liu, J. Xu and M. P. Aldred, Mater. Chem. Front., 2017, 1, 1900-1904. 
16 S.-J. Yoon, J. W. Chung, J. Gierschner, K. S. Kim, M.-G. Choi, D. Kim and S. Y. Park, J. Am. Chem. Soc., 2010, 132, 1367513683.

17 Y. Dong, B. Xu, J. Zhang, X. Tan, L. Wang, J. Chen, H. Lv, S. Wen, B. Li, L. Ye, B. Zou and W. J. Tian, Angew. Chem., Int. Ed., 2012, 51, 10782-10785.

18 X. Luo, J. Li, C. Li, L. Heng, Y. Q. Dong, Z. Liu, Z. Bo and B. Z. Tang, Adv. Mater., 2011, 23, 3261-3265.

19 H. Li, X. Zhang, Z. Chi, B. Xu, W. Zhou, S. Liu, Y. Zhang and J. Xu, Org. Lett., 2011, 13, 556-559.

20 Y. Hong, J. W. Lam and B. Z. Tang, Chem. Soc. Rev., 2011, 40, 5361-5388.

21 Z. Zhao, J. W. Lam and B. Z. Tang, J. Mater. Chem., 2012, 22, 23726-23740.

22 J. Huang, Y. Jiang, J. Yang, R. Tang, N. Xie, Q. Li, H. S. Kwok, B. Z. Tang and Z. Li, J. Mater. Chem. C, 2014, 2, 2028-2036.

23 B. Q. Liu, H. Nie, X. B. Zhou, S. B. Hu, D. X. Luo, D. Y. Gao, J. H. Zou, M. Xu, L. Wang, Z. J. Zhao, A. J. Qin, J. Peng, H. L. Ning, Y. Cao and B. Z. Tang, Adv. Funct. Mater., 2016, 26, 776-783.
24 Y. Gong, J. Liu, Y. Zhang, G. He, Y. Lu, W. B. Fan, W. Z. Yuan, J. Z. Sun and Y. Zhang, J. Mater. Chem. C, 2014, 2, 7552-7560. 25 W. Z. Yuan, X. Bin, G. Chen, Z. He, J. Liu, H. Ma, Q. Peng, B. Wei, Y. Gong and Y. Lu, Adv. Opt. Mater., 2017, 5, 1700466. 26 S. Wang, W. J. Oldham, R. A. Hudack and G. C. Bazan, J. Am. Chem. Soc., 2000, 122, 5695-5709.

27 Y. Gong, Y. Tan, J. Liu, P. Lu, C. Feng, W. Z. Yuan, Y. Lu, J. Z. Sun, G. He and Y. Zhang, Chem. Commun., 2013, 49, 4009-4011.

28 W. Z. Yuan, Y. Gong, S. Chen, X. Y. Shen, J. W. Lam, P. Lu, Y. Lu, Z. Wang, R. R. Hu, N. Xie, H. S. Kwok, Y. M. Zhang, J. Z. Sun and B. Z. Tang, Chem. Mater., 2012, 24, 1518-1528. 29 M.-J. Teng, X.-R. Jia, S. Yang, X.-F. Chen and Y. Wei, Adv. Mater., 2012, 24, 1255-1261.

30 B. Liu, W.-L. Yu, Y.-H. Lai and W. Huang, Chem. Mater., 2001, 13, 1984-1991.

31 M. R. Andersson, M. Berggren, O. Inganäs, G. Gustafsson, J. Gustafsson-Carlberg, D. Selse, T. Hjertberg and O. Wennerström, Macromolecules, 1995, 28, 7525-7529. 